\title{
Trabajo emocional y estresores laborales como predictores de ansiedad y depresión en profesores universitarios mexicanos
}

\section{Emotional work and occupational stressors as predictors of anxiety and depression in Mexican university teachers}

Fecha de recepción: 03-03-2017

Fecha de aceptación: 27-06-2018

\author{
Marlene Rodríguez-Martínez* \\ José Horacio Tovalin-Ahumada* \\ Dr. Pedro Rafael Gil-Monte \\ Universitat de València \\ Judith Salvador-Cruz* \\ Dra. Guadalupe Acle-Tomasini* \\ * Universidad Nacional Autónoma de México
}

\section{resumen/alostract:}

El objetivo de este estudio fue analizar si la condición de trabajo emocional (TE) y los estresores laborales (EL) predicen la ansiedad y depresión en un grupo de profesores universitarios mexicanos. Además, se analizó si el sexo y la edad contribuían para explicar dichos síntomas. Se estudió un grupo de 211 profesores universitarios de escuelas públicas. Los resultados mostraron que en las correlaciones y en los modelos de regresión lineal simple (stepwise) la ansiedad y la depresión son explicados por el TE y los EL. Sin embargo, en los modelos lineales múltiples, ser mujer, el TE y los EL contribuyen para predecir la ansiedad, pero solo los EL predicen la depresión en este grupo. Este estudio permite identificar variables de predicción de salud mental en profesores universitarios mexicanos, pero aún es necesario profundizar acerca de la contribución del TE y los EL sobre la salud mental de los docentes.

The purpose of this study was to analyze whether the condition of emotional work (EW) and occupational stressors (OE) predict anxiety and depression in a group of Mexican university professors. In addition, it is analyzed if sex and age contribute to explain those symptoms. A group of 211 university professors from public schools was studied. The results show that in bivariate correlations and simple linear regression models, anxiety and depression are explained by TE and OE However, in multiple linear regression models both EW and OE contribute to predict anxiety in subsample of women, but only OE predict depression in this group. This study allows the identification of mental health prediction variables in Mexican university professors, but it is still necessary to deepen the contribution of EW and OE to the mental health of teachers.

\section{palahras clave/keywords:}

Trabajo emocional, estrés laboral, ansiedad, depresión, profesores universitarios.

Emotional work, job stress, anxiety, depression, university teachers. 
A lo largo de más de 20 años, diversos autores han hecho importantes aportes para el estudio del Trabajo Emocional (TE). Variables como expresión, experiencia, variedad y tipos de actuación emocional, han contribuido con la integración de factores individuales y de la organización, para explicar al TE como un proceso de gestión organizacional (Ashforth y Humphrey, 1993; Hoschild, 1993; Morris y Feldman, 1996; Grandey, 2000; Yeong-Gyeong y Kyoung-Seok, 2015).

El estudio de la relación del TE y efectos en la salud mental, tiene como precedente investigaciones que ha establecido asociación entre la percepción emocional percibida en el trabajo y el nivel de estrés (Mann, y Cowburn, 2005) y se ha mostrado el vínculo que la salud mental mantiene con componentes estudiados del TE, como la disonancia emocional (Indregard, Knardahl y Nielsen, 2018). Sin embargo, los reportes sobre repercusiones del TE sobre indicadores de salud mental en diferentes ocupaciones, aún son escasos en la literatura científica.

En el contexto de la enseñanza, factores como las reformas educativas y condiciones de trabajo, como la elevada demanda, falta de apoyo organizacional y falta de motivación de los alumnos, han sido relacionados al desarrollo de problemáticas de salud laboral (FigueiredoFerraz, Gil-Monte, Ribeiro y Grau-Alberola, 2013; Rabasa, Figueiredo-Ferraz, Gil-Monte y Llorca-Pellicer, 2016), pero sólo las demandas emocionales, consideradas como estresores en general, son asociadas al efecto de la ansiedad y depresión en grupos de docentes (Mahan, et al., 2010; Unda et al, 2016), lo que abre una perspectiva de estudio del efecto del TE sobre indicadores de salud mental de los docentes.

\section{Trabajo emocional y docencia}

Alrededor del TE, se han propuesto diversos modelos teóricos (Nur, Topsakal e Iplik, 2014), uno de ellos, es el modelo de regulación de las emociones. Desde esta perspectiva, el TE puede ser descrito como un proceso autorregulador dinámico en relación con los clientes y los empleados (Gabriel y Diefendorff, 2015) e integra tres componentes básicos: un requisito emocional desde la organización, la regulación de las emociones en dicha relación y una expresión o comportamiento derivados de la relación cliente-empleado (Grandey y Gabriel, 2015).

En este estudio, el TE se conceptualiza como una condición laboral, que incluye los elementos básicos ya planteados desde el modelo de regulación de las emociones (Grandey, 2000; Fishbach, 2003), ajustados al entorno de trabajo del profesor universitario dentro de un contexto social de la organización (Côté, 2015). Los tres componentes del TE que se consideraron para el estudio de los profesores universitarios son: (a) tareas externas (propias de la organización y funciones docentes), (b) tareas internas (proceso de autoregulación emocional en el aula) y (c) externalización auténtica o simulada de las emociones en el contexto social la organización de las instituciones de educación superior en México (Rodríguez, Tovalín, Salvador, Gil-Monte y Acle, 2017).

\section{Trabajo emocional, ansiedad y depresión}

En poco más de 50 años de investigación se ha encontrado que variables organizacionales con un trasfondo emocional, como agotamiento, disonancia o estresores emocionales han 
sido estudiadas en relación a otros factores presentes en el trabajo (Indregard, et al., 2018; Wolter, et al, 2018), por tener un impacto potencial como predictores de la salud física y bienestar psicológico en población trabajadora (O’Driscoll y Brough, 2010). Sin embargo, el papel del TE aún no figura claramente en esa relación. Una línea importante sobre el estudio del trabajo y salud mental en docentes, ha sido la búsqueda de modelos explicativos del bienestar/malestar docente basados en aspectos psicosociales del trabajo (Cornejo y Quiñones, 2007). Desde una perspectiva individual, el procesamiento de la información emocional y la falta o déficits en el procesamiento de la recompensa, se han asociado con la presencia de ansiedad y depresión (Proudfit, Bress, Foti, Kujawa y Klein, 2015; Rottenberg, Gross, y Gotlib, 2005). Desde una perspectiva de comportamiento colectiva, Mababu (2012) ha señalado que las normas organizacionales, los requerimientos emocionales del puesto y la obligación de expresar las emociones positivas en el trabajo, también pueden influir en la expresión emocional y la salud mental.

Para explicar la relación entre el proceso emocional y el de salud-enfermedad una vía de explicación apunta a la supresión o control de emociones negativas, como las causantes de producir niveles de activación fisiológica intensa, afectando las funciones fisiológicas y deteriorando la salud si estas respuestas se cronifican (Piqueras, Ramos, Martínez y Oblitas, 2009; Pinedo, Arroyo y Caballero, 2017). Otra posibilidad de analizar esta relación es contemplar a la supresión de la expresión y experiencia emocional, como un fenómeno colectivo, que alude a un puesto, un rol o una política de empleo y que pudiera también explicar síntomas de ansiedad y depresión.

El modelo de supresión emocional puede ser una línea para explicar al TE y vincular el ambiente de trabajo con la regulación psicológica de acciones laborales y mecanismos cognitivo-emocionales que subyacen en un grupo ocupacional (Feldman y Blanco, 2006; Yeong-Gyeong y Kyoung-Seok, 2015; Zapf, 2002).

El TE en la docencia produce una exigencia cognitiva y de auto-ajuste emocional dentro del contexto de la organización (Cejudo, López-Delgado, Rubio y Latorre, 2015; Funes, 2017; Hargreaves, 2005) y aunque el TE puede parecer invisible por el rol que desempeña el maestro, permaneciendo emocionalmente disponible para los estudiantes (Maile y Feinauer, 2015), al ser una condición laboral no visible, puede derivar en emociones negativas en el aula (Katz, Harris, Abenavoli, Greenberg y Jennings, 2017), provocando un impacto sobre la salud mental, especialmente sobre síntomas de ansiedad y depresión, que causan un gran número de bajas entre los docentes (Strieder, 2009).

\section{Estresores laborales, ansiedad y depresión}

Los estresores laborales (EL), también llamados riesgos psicosociales en el trabajo, aluden a las condiciones directamente relacionadas con la organización del trabajo, el contenido del puesto, la realización de la tarea e incluso con el entorno (Gil-Monte, 2009, p. 169), que contribuyen deterioran la salud y calidad de vida laboral.

Cuando los factores psicosociales presentes en el trabajo representan una condición desfavorable para el individuo, entonces se habla de riesgos psicosociales (Gil-Monte, 2010) aluden a "aquéllos que pueden provocar trastornos de ansiedad, no orgánicos del ciclo sue- 
ño-vigilia y de estrés grave y de adaptación, derivado de la naturaleza de las funciones del puesto de trabajo, el tipo de jornada laboral y la exposición a acontecimientos traumáticos severos o a actos de violencia laboral, por el trabajo desarrollado" (Diario Oficial de la Federación, Capítulo Primero, Disposiciones generales, 2014). Desde esta perspectiva, los factores psicosociales de riesgo al presentar la probabilidad de dañar la salud física y psicológica, son negativos, y se asocian con otros factores que provienen de una relación del trabajador y sus funciones laborales.

La docencia, en el contexto mexicano, asume la responsabilidad de transmitir el conocimiento por medio del profesor, en un entorno que incluye muchas horas de sedentarismo, una buena cantidad de esfuerzo cognitivo y el involucramiento emocional fuera y dentro de la organización (Reyes, Ibarra, Torres y Razo, 2012). El profesorado universitario, es un colectivo muy heterogéneo que dedica gran parte de sus horas de trabajo en actividades de enseñanza, investigación y tareas de gestión (Vera, Sandoval y Martín, 2010); aunado a eso, enfrenta la implementación de nuevas exigencias curriculares, el incremento en la matrícula y la continua profesionalización del docente (Silas, 2017).

Algunos autores han reconocido que a causa del estrés derivado de las exigencias en el aula surgen efectos negativos en la salud física y psicológica de los docentes (Katz, et al., 2017; Viljoen y Rothmann, 2009). La depresión constituye una reacción común entre esta población y sus causas han sido atribuidas al bajo apoyo social, la demanda excesiva de trabajo y carencias tanto estructurales como didácticas, que agudiza los problemas que los docentes enfrentan en su vida laboral (Sieglin y Ramos, 2007).

Diferentes estresores organizacionales como la sobrecarga, el control y la inseguridad laboral se han asociado con la mala salud en profesores universitarios y personal escolar y se ha atribuido al grupo de mujeres, la mayor afectación por dichos estresores (Viljoen y Rothmann, 2009).

\section{Sexo, edad, ansiedad y depresión}

La ansiedad y la depresión, son condiciones de salud mental que han sido diferenciadas por sexo y edad. Se ha atribuido a las mujeres en todas las edades y a los hombres mayores de 65 años, el doble de probabilidades de dar un test positivo en problemas de salud mental, aunque estudios señalan que la edad y los años de experiencia laboral están asociados a la sintomatología mental, también se ha señalado que esta relación se desdibuja al integrar estas variables en un análisis multivariado (Galdeano, Godoy y Cruz, 2007).

Lo que se ha encontrado, es que hay evidencia sobre las diferencias neurales entre hombres y mujeres en el procesamiento de reactividad emocional y la capacidad de percibir, comprender y sentir los estados emocionales de los demás, (Derntl, et al., 2010; Filkowskia, Olsen, Duda, Wange y Sabatinelli, 2017). Las mujeres muestran una activación neuronal más fuerte en las tareas de empatía en áreas relacionadas con la emoción, se auto califican más empáticas que los varones y confían en estrategias de procesamiento donde incluyen más emociones; mientras que los hombres, utilizan áreas de procesamiento más corticales e incluyen estrategias más relacionadas con el conocimiento, (Derntl, et al., 2010). 
El objetivo principal de este trabajo fue analizar si el TE y los EL predecían el incremento de síntomas de ansiedad y depresión en un grupo de profesores universitarios. Como objetivos secundarios a este estudio se analiza si las dimensiones del TE evaluadas explican los síntomas de ansiedad y depresión, y también si el sexo y la edad contribuyen para explicar los síntomas de ansiedad y depresión en los profesores universitarios estudiados.

\section{Hipótesis}

H1. El TE y los EL son factores que explicarán la intensidad de los síntomas de ansiedad y depresión.

H2. Las dimensiones de control de emociones negativas y las normas institucionales se asociarán con un incremento en los síntomas de ansiedad y depresión.

H3. El sexo y la edad, junto con el TE y los EL integrarán un modelo que explicará el incremento de síntomas de ansiedad y depresión en profesores universitarios.

\section{Método}

\section{Participantes}

211 profesores universitarios de cuatro áreas del conocimiento completaron la evaluación en sus centros de trabajo. La distribución por sexo fue similar para hombres y mujeres (44\% y $56 \%$ respectivamente). La mayoría de los profesores tenían pareja (65.9\%) y predominó el grado de estudios de maestría de los participantes.

El área donde se encontró mayor participación fue la de Ciencias Biológicas, Químicas y de la Salud (39\%), la mitad de ellos tenían una plaza de trabajo definitiva y eran de asignatura que, de acuerdo con estatutos institucionales, su principal función es la impartición de clases y no la investigación y todos pertenecía a universidades públicas ubicadas en la Ciudad de México y área metropolitana.

Se utilizó un diseño no experimental transversal de grupo único (Montero y León, 2005) cuyos criterios de inclusión fue ser profesor universitario, aceptar participar bajo consentimiento informado, completar la evaluación por medio de cuestionario y tener al menos un año en labores docentes. Los profesores en promedio trabajan 18 horas frente a grupo, imparten tres materias anuales y/o semestrales y atienden poco más de 80 alumnos por profesor.

\section{Instrumentos}

Ficha de identificación. $\mathrm{Al}$ inicio de los cuestionarios que se aplicaron se acompañó con datos de identificación sociodemográfica de sexo, edad, nivel de estudios y estado civil. También se incluyeron datos de identificación laboral: antigüedad, horas frente a grupo, número de asignaturas, número de alumnos atendidos, que fueron un referente para contextualizar la demanda de trabajo del profesor universitario.

Trabajo emocional. Se utilizó la escala para la evaluación del Trabajo Emocional de Profesores de Educación Superior (TEES), que se construyó y validó para el uso en docentes universitarios mexicanos. Cuenta con 15 reactivos tipo Likert y cinco opciones de respuesta 
que van de Siempre a Nunca (5 a 1 respectivamente). La escala TEES fue sometida a un análisis factorial exploratorio, obteniendo un valor adecuado de contraste para sus reactivos $(\mathrm{KMO}=0.953, \mathrm{p}=0.00)$. Se identificaron cuatro componentes finales con adecuado valor de alfa $(\alpha=.81)$ y que explicaron el $61.4 \%$ de la varianza total (Rodríguez, et al., 2017).

El factor de Normas Autoimpuestas, integrado por tres reactivos, agrupa los comportamientos que los profesores universitarios asumen en el aula como parte de sus funciones $(\alpha=.72)$. El factor Control de Emociones Negativas se compone de cuatro reactivos y agrupa conductas que el profesor evita en el trato con los alumnos $(\alpha=.71)$. El factor Expresión Forzada de Emociones Positivas se compone de cinco reactivos y agrupa conductas positivas que el profesor muestra con los alumnos, pero que no son las que realmente siente $(\alpha=.72)$. Y el factor de Normas Institucionales integra tres reactivos que agrupa los comportamientos que la institución dicta para la relación alumno-maestro $(\alpha=.72)$. Para la puntuación de cada dimensión, se suman los reactivos que la agrupan, teniendo como valor mínimo 1 y como valor máximo 15, 20 ó 25, según sea el total de reactivos que agrupa. Para el puntaje total, se suman los puntajes de todas las dimensiones. En el análisis de resultados de este estudio, se tomó el valor de la sumatoria de cada dimensión y la sumatoria total, y en la descripción de resultados se muestra el promedio de cada dimensión obtenida para el grupo de estudio.

Estresores laborales. La escala para valorar los EL se trata de un instrumento que evalúa estresores en el trabajo de profesores universitarios mexicanos, consta de 13 reactivos, 10 de ellos tomados de Unda et al. (2016) que miden sobrecarga de trabajo e inequidad y tres reactivos más que evalúan apoyo de compañeros, tomado de Téllez (2015).

La escala fue sometida a un análisis factorial exploratorio, obteniendo un valor aceptable de contraste para sus reactivos $(\mathrm{KMO}=0.792, \mathrm{p}=0.00)$. Se identificaron tres componentes finales con adecuado valor de alfa $(\alpha=.77)$, que explicaron el $53.7 \%$ de la varianza total y se eliminaron 2 de los 9 reactivos originales que medían inequidad, de modo que los factores finales fueron sobrecarga de trabajo (4 reactivos), inequidad (6 reactivos) y apoyo de compañeros (3 reactivos). Para el análisis de los resultados de este estudio se tomó el puntaje total de los estresores propuestos divididos entre el número total de reactivos.

Depresión y ansiedad. Escala Kessler 10 (K-10). Esta escala es una prueba de tamizaje usada para la detección de depresión y ansiedad en el primer nivel de atención. Ha sido validada para población mexicana sana y con trastornos de ansiedad y depresión, presentando una alta consistencia interna $(\alpha=.90)$. Tanto la escala de Ansiedad $(\alpha=.81)$ como la de Depresión $(\alpha=.80)$ presentaron en este estudio valores adecuados de fiabilidad. Esta escala utiliza los criterios del Eje I del DSM-IV y la CIE-10 para la confirmación del diagnóstico de depresión y de ansiedad, no obstante, sus autores consideran que su clasificación debe usarse con el fin de identificación, no para tratamiento y/o seguimiento de personas con estos trastornos (Vargas, Villamil, Rodríguez, Pérez y Cortés, 2011).

\section{Procedimiento}

La recogida de datos se realizó directamente en los centros de trabajo de los profesores previa invitación y notificación de consentimiento informado, que fue firmado directamente en un cuadernillo donde se integró una batería con los instrumentos de evaluación. 
Para analizar los datos se empleó el programa estadístico SPSS en su versión 21. El punto de corte para la significación estadística fue establecido en $\mathrm{p}<.05$. La descripción de las características laborales y puntajes de las variables TE, EL, síntomas de ansiedad y síntomas de depresión se efectuaron empleando estadísticos descriptivos de media y desviación estándar. Para identificar la asociación entre las variables de TE, EL, síntomas de ansiedad y síntomas de depresión se utilizó el análisis de correlación bivariada. Para el análisis multivariado, inicialmente se corrió un modelo de regresión lineal de TE para síntomas de ansiedad y depresión y otro modelo de EL para síntomas de ansiedad y depresión. Posteriormente, se controló el efecto del sexo y edad en un modelo de regresión lineal múltiple con el método jerárquico para identificar si se mantenía el efecto de las variables independientes, o si el efecto sobre los síntomas de ansiedad y depresión se desdibujaba en el grupo de profesores universitarios.

\section{Resultados}

Los promedios del puntaje de TE y sus subescalas, EL, Ansiedad y Depresión se muestran en la Tabla 1, siendo mayor la media para Ansiedad, comparándola con la media de Depresión en los mismos docentes.

Tabla 1. Descriptivos de las variables para la muestra del estudio.

\begin{tabular}{lcccc}
\hline & M & $d t$ & Máx & Alfa \\
\hline Trabajo emocional & 56.4 & 12.9 & 75 & .81 \\
Normas institucionales & 10.4 & 2.7 & 15 & .72 \\
Normas autoimpuestas & 12.6 & 2.1 & 15 & .72 \\
Control de emociones negativas & 14.9 & 3.4 & 20 & .71 \\
$\quad$ Expresión forzada de emociones positivas & 19.4 & 3.6 & 25 & .72 \\
Estresores laborales & 1.8 & .59 & 4 & .77 \\
Ansiedad & 9.4 & 3.5 & 25 & .81 \\
Depresión & 6.8 & 2.8 & 25 & .80 \\
\hline
\end{tabular}

Los resultados del análisis de correlaciones entre TE, sus subescalas y los puntajes de EL, Ansiedad y Depresión se muestran en la Tabla 2. Puede apreciarse que el TE se asocia positiva y significativamente con los EL, Ansiedad y Depresión, siendo mayor la asociación entre TE y síntomas de ansiedad. De las subescalas de TE, el Control de Emociones Negativas y la Expresión Forzada de Emociones Positivas, se asocian positiva y significativamente sólo con el puntaje de Ansiedad. Los EL se asocian positiva y significativamente tanto con el puntaje de Ansiedad y Depresión, pero negativamente con las Normas Institucionales.

El modelo de regresión lineal simple para TE se presenta en la Tabla 3. Muestra que el modelo lineal fue significativo, tanto para los síntomas de Ansiedad como de Depresión, con varianzas explicadas entre 5\% y 10,8\%. El modelo de regresión lineal simple para EL se presenta en la Tabla 4. Muestra que el modelo lineal fue significativo para los síntomas 
Tabla 2.- Correlación entre Trabajo emocional y sus dimensiones, Estresores laborales, Ansiedad y Depresión.

\begin{tabular}{|c|c|c|c|c|c|c|c|c|}
\hline & 1 & 2 & 3 & 4 & 5 & 6 & 7 & 8 \\
\hline 1. TE & 1 & & & & & & & \\
\hline 2. NI & $.72^{* *}$ & 1 & & & & & & \\
\hline 3. NA & $.76^{* *}$ & $.42^{* *}$ & 1 & & & & & \\
\hline 4. CEN & $.83^{* *}$ & $.46^{* *}$ & $.51^{* *}$ & 1 & & & & \\
\hline 5. EFEP & $.85^{* *}$ & $.51^{* *}$ & $.54^{* *}$ & $.66^{* *}$ & 1 & & & \\
\hline 6. EL & $.25^{* *}$ & $-.30^{* *}$ & -.02 & .05 & .01 & 1 & & \\
\hline 7. ANS & $.34^{* *}$ & .01 & .07 & $.17^{*}$ & $.15^{*}$ & $.41^{* *}$ & 1 & \\
\hline 8. DEP & $.25^{* *}$ & .05 & -.02 & .10 & .10 & $.26^{* *}$ & $.73^{* *}$ & 1 \\
\hline
\end{tabular}

Nota. 1. TE, Trabajo emocional; 2. NE, Normas institucionales; 3. NA, Normas autoimpuesta; 4. CEN, Control de emociones negativas; 5. EFEP. Expresión forzada de emociones positivas; 6 . Estresores laborales; 7. Ansiedad; 8. Depresión.

$$
* * p<.01, * p<.05
$$

de Ansiedad y Depresión, con varianzas explicadas entre 6,2\% y 16,8\%. El modelo con las subescalas del TE no fue significativo para los síntomas de Ansiedad ni Depresión. No obstante, de las subescalas estudiadas sólo el control de Emociones Negativas, $\left(\beta_{\text {adj }}=.163, g l=\right.$ $4, p=.097$ ) podría contribuir a explicar el incremento de síntomas de Ansiedad.

Tabla 3.- Análisis de regresión de las variables Ansiedad y Depresión sobre Trabajo emocional.

\begin{tabular}{|c|c|c|c|c|}
\hline \multicolumn{5}{|c|}{ Ansiedad } \\
\hline & beta & $\mathrm{t}$ & $\mathrm{R}_{\mathrm{adj}}^{2}$ & $\mathrm{~F}_{(1,211)}$ \\
\hline Trabajo emocional & $.34 * * *$ & $5.131 * * *$ & .108 & \\
\hline Estadístico ecuación & & & & $26.33 * * *$ \\
\hline \multicolumn{5}{|c|}{ Depresión } \\
\hline & beta & $\mathrm{t}$ & $\mathrm{R}_{\mathrm{adj}}^{2}$ & $F_{(1,211)}$ \\
\hline Trabajo emocional & $.25 * * *$ & $3.49 * * *$ & .05 & \\
\hline Estadístico ecuación & & & & $13.69 * * *$ \\
\hline
\end{tabular}

Se generaron dos modelos de regresión múltiple con el método de regresión jerárquica. Uno para Ansiedad y otro para Depresión, donde se introdujeron las variables TE, EL, sexo, creando una variable dummy para mujer y edad. El modelo de regresión lineal múltiple para síntomas de Ansiedad mostró significancia estadística, aportando el 15,3\% de la varianza explicada $\left(\mathrm{R}^{2}=.153, g l=3, p<.000\right)$. Este modelo muestra que todas las variables contribuyen para explicar el incremento del puntaje de ansiedad, excepto la edad (Tabla 5). El 
Tabla 4.- Análisis de regresión de las variables Ansiedad y Depresión sobre Estresores laborales.

\begin{tabular}{|c|c|c|c|c|}
\hline \multicolumn{5}{|c|}{ Ansiedad } \\
\hline & beta & $\mathrm{t}$ & $\mathrm{R}_{\mathrm{adj}}{ }^{2}$ & $F_{(1,211)}$ \\
\hline Trabajo emocional & $41 * * *$ & $6.96 * * *$ & .168 & \\
\hline Estadístico ecuación & & & & $43.32 * * *$ \\
\hline \multicolumn{5}{|c|}{ Depresión } \\
\hline & beta & $\mathrm{t}$ & $\mathrm{R}_{\mathrm{adj}}{ }^{2}$ & $F_{(1,211)}$ \\
\hline Trabajo emocional & $26 * * *$ & $3.86 * * *$ & .062 & \\
\hline Estadístico ecuación & & & & $14.91 * * *$ \\
\hline
\end{tabular}

modelo de regresión lineal múltiple con el método de regresión jerárquica para Depresión mostró significancia estadística, aportando el $1,7 \% \%$ de la varianza explicada $\left(\mathrm{R}_{\mathrm{adj}}{ }^{2}=.017\right.$, $g l=3, p<.000)$, excluyendo del análisis las variables TE, mujer y edad, excepto los EL $\left(\beta_{a d j}\right.$ $=.017, p=.034$ ), que contribuyen con el incremento del puntaje de depresión (Tabla 5).

Tabla 5.- Análisis de regresión múltiple jerárquica de las variables Ansiedad y Depresión.

\begin{tabular}{|c|c|c|c|c|}
\hline \multicolumn{5}{|c|}{ Ansiedad } \\
\hline & beta & $\mathrm{t}$ & $\mathbf{R}_{\text {adj }}^{2}$ & $\mathrm{~F}_{(3,211)}$ \\
\hline Trabajo emocional & $.18^{* * *}$ & $2.64 * * *$ & & \\
\hline Estresores laborales & $.31 * * *$ & $4.59 * * *$ & & \\
\hline Mujer & $.18^{* * *}$ & $2.64 * * *$ & & \\
\hline Estadístico ecuación & & & .153 & $12.68 * * *$ \\
\hline \multicolumn{5}{|c|}{ Depresión } \\
\hline & beta & $\mathrm{t}$ & $\mathrm{R}_{\mathrm{adj}}{ }^{2}$ & $\mathrm{~F}_{(1,211)}$ \\
\hline Estresores laborales & $.15^{*}$ & $2.08^{*}$ & .017 & \\
\hline Estadístico ecuación & & & & $4.31 *$ \\
\hline
\end{tabular}

\section{Discusión}

El objetivo principal de este estudio fue analizar si la condición de TE y EL predicen el incremento de síntomas de ansiedad y depresión en un grupo de profesores universitarios y los hallazgos de este estudio muestran que hay relación tanto de una condición estresante, como de una emocionalmente exigente, con el incremento en la intensidad de síntomas de ansiedad y depresión en profesores universitarios.

Estudios como los de Mérida-López, Extremera y Rey (2017) han señalado a los factores estresantes en el trabajo como causantes del desajuste psicológico y la mala salud mental de los docentes y en el caso del TE se indica que la supresión o manifestación de emociones, tiene efectos diferentes para la salud del trabajador. Particularmente, en el ámbito docente, 
el TE sólo se ha asociado individualmente con la presencia de burnout, el cual se ha asociado con la supresión crónica de pensamientos y la inteligencia emocional percibida (Extremera, Fernández-Berrocal y Durán, 2003). A nivel organizacional el TE, se ha asociado con el desempeño en el aula, no obstante, este estudio muestra también la posibilidad de que incremente la intensidad de síntomas de ansiedad en el grupo de profesores universitarios.

Uno de los objetivos secundarios de este estudio, fue identificar si las dimensiones del TE evaluadas explicaban los síntomas de ansiedad y depresión, esperando que el control de las emociones negativas y las normas institucionales incrementaran los síntomas de ansiedad y depresión. Encontrando que estas dimensiones se asociaron con el incremento de síntomas de ansiedad en el análisis de correlación, pero los resultados del modelo de regresión no permiten señalar que dichos factores puedan predecir el incremento de síntomas de ansiedad y depresión para este grupo de profesores universitarios. Yin, Huang y Lee (2017) han señalado que variables individuales como la regulación de las emociones contribuyen de forma importante para predecir los puntajes de los docentes sobre los síntomas depresivos, ansiosos y de estrés, en este estudio, sólo el control de emociones negativas indicó una posibilidad de esa relación.

El último objetivo planteado en este estudio fue identificar si el sexo y la edad contribuían para explicar los síntomas de ansiedad y depresión en un modelo multidimensional. Algunos trabajos han demostrado que no existen diferencias significativas desde el punto de vista del género ni de la edad en cuanto al control de las emociones se refiere, y tampoco hay diferencias significativas sobre el bienestar psicológico (Erikson y Ritter, 2001; Gray, 2009; Sohn y Lee, 2012). Los resultados de este estudio muestran que ser mujer, el TE y los EL, en un modelo multidimensional predicen la intensidad de los síntomas de ansiedad, mas no la depresión y este resultado coincide con otros trabajos (Rey, Extremera y Pena, 2016; Mérida-López, Quintana-Orts, Extremera y Rey-Peña, 2017) que han señalado el papel del sexo, junto las habilidades emocionales, como mediadores que explican el estrés en el trabajo.

\section{Limitaciones}

La evaluación del TE, aunque permitió identificar la intensidad de emociones percibidas y una valencia emocional negativa en el contexto social de la organización del docente mexicano, se asoció únicamente con los síntomas de ansiedad, mientras que los EL estudiados permitieron evaluar su efecto sobre los síntomas de ansiedad y depresión en docentes universitarios.

Hay que estudiar con mayor profundidad la valencia negativa del TE e incorporar la valencia positiva de éste, así como otros factores colectivos e institucionales que pueden mediar el efecto sobre la salud mental del docente, pues otros autores han señalado que la falta de recursos individuales del profesor puede llevarlo al límite físico, emocional y psicosocial (Arévalo-Pachón y Guerrero, 2010; Martínez, 2009).

\section{Futuras reflexiones}

La literatura ha demostrado un papel mediador de la regulación emocional y la exposición a estresores de origen laboral en el desarrollo de depresión y enfermedad física y mental 
(Compare, Zarbo, Shonin, Van Gordon y Marconi, 2014), sin embargo, en el caso del TE los resultados aún no son consistentes.

Considerar que el TE del docente puede dividirse sólo en algunas estrategias de actuación, sería ilusorio, debido a que no todas las expresiones se autoregulan como un mecanismo consciente (Grandey y Gabriel, 2015) y es posible que no exista un único perfil en riesgos psicosociales del profesor universitario.

Aunque las altas cargas de trabajo y el constante manejo de la conducta de los alumnos dentro del aula o la dificultad de las materias impartidas sea un común denominador de la profesión (González, Landeros y Ruiz, 2008; Mazer, McKenna-Buchanan, Quinlan Titswort, 2014), todavía hay que pensar en estudios cualitativos y longitudinales que puedan dar cuenta del fenómeno de las emociones en el ámbito del profesor universitario y su relación con la salud del docente, sobre todo porque el efecto negativo de condiciones emocionalmente estresantes o altamente demandantes en el trabajo han probado su relación con la mala salud y el deterioro de la calidad de vida a causa de ésta (Phillips, Sen y McNamee, 2007; Wong, Poole y Agius, 2015), y así contribuir con líneas de intervención efectiva para esta población en riesgo.

\section{Referencias}

Arévalo-Pachón, G. y Guerrero, J. (2012). Condiciones de trabajo, estrés y salud autorreportadas de los trabajadores docentes y no docentes de una institución privada de educación superior. Tesis Psicológica, 5(1), 76-97.

Ashforth B.E., Humphrey R.H. (1993). Emotional labor in service roles: The influence of identity. Academy of Management Review, 18, 88-115.

Cejudo, J., López-Delgado, M.L., Rubio, M.J. y Latorre, J.M. (2015). La formación en educación emocional de los docentes: una visión de los futuros maestros. Revista Española de Orientación y Psicopedagogía, 26(3), 45-62.

Compare, A., Zarbo, C., Shonin, E., Van Gordon, W. y Marconi, C. (2014). Emotional regulation and depression: A potentialmediator between heart and mind. Cardiovascular Psychiatry and Neurology, 2014 (6). doi: $10.1155 / 2014 / 324374$

Cornejo, R. y Quiñónez, M. (2007). Factores asociados al malestar/bienestar docente. Una investigación actual. Revista Iberoamericana sobre Calidad, Eficacia y Cambio en Educación, 5(5), 75-80.

Côté, S. (2015). A social interaction model of the effects of emotion regulation on work strain. Academy of Management, 30(3), 509-530.

Derntl, B., Finkelmeyer, A., Eickhoff, S., Kellermann, T., Falkenberg, D.I., Schneider, F., et al. (2010). Multidimensional assessment of empathic abilities: neural correlates and gender differences. Psychoneuroendocrinology 35, 67-82.

Diario Oficial de la Federación (2010). Capítulo Primero, Disposiciones generales, México, 13 de noviembre de 2010. Disponible en: http://www.dof.gob.mx/nota_detalle.php?codigo $=5368114 \&$ fecha $=13 / 11 / 2014$

Erickson, R. y Ritter, C. (2001). Emotional Labor, Burnout and in authenticity. Does Gender Matter? Social Psychology Quartely, 64, 146-163.

Extremera, N., Fernández-Berrocal, P. y Durán, E. (2003). La inteligencia emocional y burnout en profesores. Encuentros en Psicología Social, 1, 260-265. 
Feldman, L. y Blanco, G. (2006). Las emociones en el ambiente laboral: un nuevo reto para las organizaciones. Revista de la Facultad de Medicina, 29(2), 103-108.

Figueiredo-Ferraz, H., Gil-Monte, P.R., Ribeiro, B. y Grau-Alberola, E. (2013). Influence of a cognitive behavioural training program on health: A study among primary teachers. Revista de Psicodidáctica, 18(2), 341-354. doi: 10.1387/RevPsicodidact.6995

Filkowskia, M.M., Olsen, R.M., Duda, B., Wange, T.J. y Sabatinelli, D. (2017). Sex differences in emotional perception: Meta analysis of divergent activation. Neurolmage, 147, 925-933. doi: 10.1016/j.neuroimage.2016.12.016

Fischbach, A. (2003), "Determinants of emotion work". Disertación para obtener el doctorado de la Facultad de Matemáticas y Ciencias Naturales de la Georg-August-Universität zu Göttingenavailable. Disponible en: https://www.researchgate.net/profile/Andrea_Fischbach/publication/35902026_Determinants_of_emotion_work_Elektronische_Ressource/links/558955f408ae2affe71481f8.

Funes, S. (2017). Las emociones en el profesorado: el afecto y el enfado como recursos para el disciplinamiento. Educação e Pesquisa, 43(3), 785-798. doi: 10.1590/S1517-702201610149719

Gabriel, A.S. y Diefendorff, J.M. (2015). Emotional Labor Dynamics: A Momentary. Academy of Management Journal, 58(6), 1804-1825. doi: 10.5465/amj.2013.1135

Galdeano, H., Godoy, P. y Cruz, I. (2007). Factores de riesgo psicosocial en profesores de educación secundaria. Archivos de Prevención de Riesgos Laborales, 10(4), 174-180.

Gil-Monte, P.R. (2009). Algunas razones para considerar los riesgos psicosociales en el trabajo y sus consecuencias en la salud pública. Revista Española de Salud Pública, 83 (2), 169-173.

Gil-Monte, P. R. (2010). Situación actual y perspectiva de futuro en el estudio del estrés laboral: la Psicología de la Salud Ocupacional. Informació Psicològica, 100,68-83.

González, M.T., Landero R., Ruiz, M.A. (2008). Modelo estructural predictor de la salud mental y física en mujeres. Revista Panamericana de Salud Pública, 23(2),101-108.

Grandey, A.A. (2000). Emotional regulation in the workplace: A new way to conceptualize emotional labor. Journal of Occupational Health Psychology, 5(1), 95-110.

Grandey, A.A y Gabriel, A.S. (2015). Emotional labor at a crossroads: Where do we Go from here? Annual Review of Organizational Psychology and Organizational Behavior. 2, 323-349. doi: 10.1146/annurev-orgpsych-032414-111400

Gray, B. (2009). The emotional labour of nursing. Defining and managing emotions in nursing work. Nurse Education Today, 29, 168-175.

Hargreaves, A. (2005). The geographies of teaching. Teacher College Record, 103(6), 1056-1080.

Hochschild, A.R. (1983). The managed heart. The comerzialitation of human feeling. Berkeley, CA, US: University of California Press.

Indregard, A.M., Knardahl, S. y Nielsen, M.B. (2018). Emotional dissonance, mental health complaints, and sickness absence among health- and social workers. The moderating role of self-efficacy. Frontiers in Psychology, 9(592), 1-9. doi: 10.3389/fpsyg.2018.00592

Katz, D.A., Harris, A., Abenavoli, R., Greenberg, M.T y Jennings, P.A. (2017). Educators' emotion regulation strategies and their physiological indicators of chronic stress over 1 year. Stress \& Health, 34(2), 278-285. doi: 10.1002/ smi.2782

López-Vílchez, J. \& Gil-Monte, P.R (2015). Sobrecarga laboral y de gestión del personal docente en el entorno universitario actual en España. Arxius de Ciències Socials, 32, 111-120.

Mababu, R. (2012). El constructo de Trabajo Emocional y su relación con el Síndrome del Desgaste Profesional. International Journal of Psychology and Psychological Therapy, 12(2), 219-244.

Mahan, P.L., Mahan, M.P., Park, N.J., Shelton, C., Brown, K.C y Weaver, M.T. (2010). Work environment stressors, social support, anxiety, and depression among secondary school teachers. American Association of Occupational Health Nurses Journal, 58(5), 197-205. 
Maile, R. y Feinauer, E. (2015). The emotional work of discomfort and vulnerability in multicultural teacher education. Journal of Teachers and Teaching. Theory and Practice, 21(88), 1010-1025. doi: 10.1080/13540602.2015.1005869

Mann, S. y Cowburn, J. (2005). Emotional labour and stress within mental health nursing. Journal of Psychiatric and Mental Health Nursing, 12, 154-162. doi: 10.1111/j.1365-2850.2004.00807.x7

Martínez, C.A. (2009). La docencia: enfermedades frecuentes en esta profesión. Revista Académica Semestral: Cuadernos de Educación y Desarrollo, 1(1). Disponible en: http://www.eumed.net/rev/ced/02/cam6.htm

Mazer, J.P., McKenna-Buchanan, T.P., Quinlan, M.M. y Titswort, S. (2014). The dark side of emotion in the classroom: Emotional processes as mediators of teacher communication behaviors and student negative emotions. Journal Communication Education, 63(3), 149-168. doi: 10.1080/03634523.2014.904047

Mérida-López, S., Extremera, N. y Rey, L. (2017). Emotion-regulation ability, role stress and teachers' mental health. Occupational Medicine, 67(7), 540-545. doi: 10.1093/occmed/kqx125

Mérida-López, S., Quintana-Orts, C., Extremera, N., y Rey-Peña, L. (2017). Dealing with teachers' role stressors and depressive symptomatology: does gender impact on the buffering effect of emotion regulation ability? Disponible en: http://hdl.handle.net/10630/14331

Montero, I. y León, 0.G. (2005). Sistema de clasificación del método en los informes de investigación en Psicología. Internacional. Journal of Clinical and Health Psychology, 5, 115- 127.

Morris, J.A. y Feldman, D.C. (1996). The dimensions, antecedents and consequences of emotional labour. Academy of Management, 21, 986-1010

Nur, F., Topsakal, Y. y Iplik, E. (2014). The effects of emotional labor on job attitudes of hotel employees: Mediating and moderating roles of social support and job autonomy. International Review of Management and Marketing, 4(3), 175-186.

O'Driscoll, M.P., y Brough, P. (2010). Work organization and health. En S. Leka, y J. Houdmont (Eds), Occupational Health Psychology. (pp. 57-87). Wiley-Blackwell.

Phillips, S., Sen, D. y McNamee, R. (2007). Prevalence and causes of self-reported work-related stress in head teachers. Occupational Medicine, 57(5), 367-376. doi: 10.1093/occmed/kqm055

Pinedo, R., Arroyo, M.J y Caballero, C. (2017). Afectividad positiva y negativa en el futuro docente. Revista de Educación, 20, 11-16.

Piqueras, J., Ramos, V., Martínez, A. y Oblitas, L. (2009). Emociones negativas y su impacto en la salud mental y física. Suma Psicológica, 16(2), 85-112.

Proudfit, G.H., Bress, J.N., Foti, D., Kujawa, A., y Klein, D.N. (2015). Depression and event-related potentials: Emotional disengagement and reward insensitivity. Current Opinion in Psychology, 4, 110-113. doi: 10.1016/j.copsyc.2014.12.018

Rabasa, B., Figueiredo-Ferraz, H., Gil-Monte, P.R. y Llorca-Pellicer, M. (2016). The role of guilt in the relationship between teacher's job burnout syndrome and the inclination toward absenteeism. Revista de Psicodidáctica, 21(1), 103-119. doi: 10.1387/RevPsicodidact.13076

Rey, L., Extremera, N., y Pena, M. (2016). Emotional competence relating to perceived stress and burnout in Spanish teachers: a mediator model. Psychiatry and Psychology, 2-14. doi: 10.7717/peerj.2087

Reyes, L., Ibarra, D., Torres, M. y Razo, R. (2012). El estrés como un factor de riesgo en la salud: análisis diferencial entre docentes de universidades públicas y privadas. Revista Digital Universitaria, 13(7), 3-14.

Rodríguez, M., Tovalin, J.H., Salvador, J., Gil-Monte, P.R. y Acle, G. (2017). Trabajo emocional asociado con ansiedad, depresión y marcadores biológicos en profesores universitarios. Manuscrito no publicado, Universidad Nacional Autónoma de México, Especialización en Salud en el trabajo. México. Proyecto PAPIIT 300-315.

Rottenberg, J., Gross, J.J., y Gotlib, I.H. (2005). Emotion context insensitivity in major depressive disorder. Journal of Abnormal Psychology, 114, 627-639.

Sieglin, V. y Ramos, M.E. (2007). Estrés laboral y depresión entre maestros del área metropolitana de Monterrey. Revista Mexicana de Sociología, 69(3), 517-551. 
Silas, J. (2017). Una mirada a los fenómenos cíclicos presentes en el sistema educativo superior mexicano. Revista de la Educación Superior, 46(181), 105-108. doi: 10.1016/j.resu.2017.01.001

Sohn, H.K. y Lee T.J. (2012). Relationship between HEXACO personality factors and emotional labour of service providers in the tourism industry. Tourism Management, 33, 116-125.

Strieder, R. (2009). Depressão e ansiedade em profissionais da educação das regiões da Amerios e da AMEASC. Roteiro, 34(2), 243-268.

Téllez, J. (2015). Construcción de una escala para evaluar factores de riesgo psicosocial en el trabajo con profesores de educación medio superior del d.f. Tesis de licenciatura. Universidad Nacional Autónoma de México, México.

Unda, S., Uribe, F., Jurado, S., García, M., Tovalin, H. y Juárez, A. (2016). Elaboración de una escala para valorar los factores de riesgo psicosocial en el trabajo de profesores universitarios. Journal of Work and Organizational Psychology, 32(2), 67-74. doi: 10.1016/j.rpto.2016.04.004

Vargas, B.E., Villamil, V., Rodríguez, C., Pérez, J., y Cortés, J. (2011). Validación de la escala Kessler 10 K-10 en la detección de depresión y ansiedad en el primer nivel de atención. Propiedades psicométricas. Salud Mental, 34 23-331.

Vera, M., Sandoval, M. y Martín, B. (2010). University faculty and work-related well-being: the importance of the triple work profile. Electronic Journal of Research in Educational Psychology, 8(21) 581-602.

Viljoen, J.P. y Rothmann, S. (2009). Occupational stress, ill health and organisational commitment of employees at a university of technology. Journal of Industrial Psychology, 35(1), 67-77.

Wolter, C., Santa Maria, A., Wörfel, F., Gusy, B., Lesener, T., Kleiber, D. y Renneberg, B. (2018). Job demands, job resources, and well-being in police officers -a resource-oriented approach. Journal of Police and Criminal Psychology, 1-10.

Wong, M.G., Poole, C.J. y Agius. R. (2015). Attribution of mental illness to work: a Delphi study. Occupational Medicine, 65(5), 391-397. doi: 10.1093/occmed/kqv050

Yeong-Gyeong, Ch. y Kyoung-Seok, K. (2015). A literature review of emotional labor and emotional labor strategies. Universal Journal of Management, 3, 283-290. doi: 10.13189/ujm.2015.030704

Yin, H., Huang, S y Lee, J.C. (2017). Choose your strategy wisely: Examining the relationships between emotional labor in teaching and teacher efficacy in Hong Kong primary schools. Teaching and Teacher Education, 66, 127-136.

Zapf, D. (2002). Emotion work and psychological well-being A review of the literature and some conceptual considerations. Human Resource Management Review, 12, 237-268. 